\title{
Thoracic manifestation of Wegener's granulomatosis: Computed tomography findings and analysis of misdiagnosis
}

\author{
JIAKAI LI ${ }^{1,2}$, CHUANGUI LI ${ }^{3}$ and JIAOJIAO LI ${ }^{4}$ \\ ${ }^{1}$ Department of Radiology, The Hainan Branch of The Chinese PLA General Hospital, Sanya, Hainan 572013; \\ ${ }^{2}$ Department of Radiology, The Chinese PLA General Hospital, Beijing 100853; ${ }^{3}$ Department of Radiology, \\ The First Affiliated Hospital, Northern Hebei College, Zhangjiakou, Hebei 075000; ${ }^{4}$ Department of Radiology, \\ The General Hospital, Tianjin Medical University, Tianjin, Hebei 300052, P.R. China
}

Received August 9, 2017; Accepted January 18, 2018

DOI: $10.3892 /$ etm.2018.6154

\begin{abstract}
The aim of the present study was to investigate the computed tomography (CT) manifestations of Wegener granulomatosis (WG) in the chest and potential reasons for misdiagnosis. Conventional CT scans and clinical data of 45 patients with WG were retrospectively analyzed. Patients typically presented with multiple system involvement, primarily in the upper and lower respiratory tract. The incidence of thoracic involvement was $75.56 \%$ (34/45). Radiographic features were varied between cases in the present study, with the most common features being numerous cavitary nodules and masses in the lungs. Cavitations were usually irregular, with uneven wall thickness, partial centrality, fuzzy inner edges and piecemeal necrosis. These results indicate that WG typically has multiple system involvement, with the chest being most prominent. Multiple variable-sized cavitary nodules with irregular edges and piecemeal necrosis were the most notable features revealed using CT scanning; however, in order to give a definitive diagnosis, biopsies should be performed.
\end{abstract}

\section{Introduction}

Wegener's granulomatosis (WG) was first described by German pathologist Dr Friedrich Wegener as rhinogenic granulomatosis in 1936 (1) and is an uncommon vasculitis of small and medium-sized arteries (2). WG, which is an angiogenic and multiple system necrotizing disease involving the upper and lower respiratory tract and kidneys (3-6), is affected by a number of factors, including heredity, infection, the immune system and the environment, and diagnosis is

Correspondence to: Dr Jiakai Li, Department of Radiology, The Hainan Branch of The Chinese PLA General Hospital, 1st Floor Outpatient Building, Jianglin Road, Haitang, Sanya, Hainan 572013, P.R. China

E-mail: lijiakai2004@aliyun.com

Key words: Wagener's granulomatosis, tomography, X-ray computed, diagnostic errors typically confirmed via clinic and laboratory examinations (7). Exposure to environmental agents, including cadmium (8), silica, mercury, sand dust and volatile hydrocarbons, has been reported to lead to the development of WG; however, no single causative agent has been identified (9). Modern immunosuppressive treatment methods greatly improve patient outcomes, with the estimated median survival time increasing to 21.7 years post-diagnosis (10). Patients may develop WG at any age, with the mean age at diagnosis being 50 year, and males and females are equally affected (11). The majority $(\sim 90 \%)$ of clinically apparent cases are reported in Caucasian individuals (11).

The thorax is commonly involved in WG and, as such, the majority of patients present with pulmonary or upper respiratory tract involvement (12). Due to its high sensitivity for detecting pulmonary involvement, computed tomography (CT) is primarily used to examine the chest in patients with WG (13-15). However, since certain characteristics of WG are difficult to detect using clinical, laboratory and imaging examination it is frequently misdiagnosed.

In the present study, the clinical manifestations, CT results and diagnosis of 45 patients with diagnosed WG were retrospectively reviewed. The aim of the present study was to identify ways of decreasing the rate of misdiagnosis and improving the diagnostic accuracy of WG involving the chest with CT.

\section{Materials and methods}

Patients. A total of 45 patients with clinically confirmed WG were selected retrospectively using a computerized search of records from patients who were treated at the Chinese PLA General Hospital (Beijing, China) between January 2011 and June 2013. The patients, including 24 men and 21 women, were 19-74 years old (mean, 46 \pm 12.2 years). The clinical symptoms were as follows: Fever accompanied with or without fatigue $(n=26,26 / 45 ; 57.78 \%)$, night sweats $(n=2,2 / 45 ; 4.44 \%)$, weakness $(n=2,2 / 45 ; 4.44 \%)$, abnormal myelogram $(n=29$, $29 / 45 ; 64.44 \%)$, cough $(n=25,25 / 45 ; 55.56 \%)$ and expectoration $(n=15,15 / 45 ; 33.33 \%)$. A total of 32 patients underwent screening examination with antineutrophil cytoplasmic antibody (ANCA) and a total of 28 patients received biopsy, 
including a CT-guided percutaneous transthoracic lung biopsy $(\mathrm{n}=10)$, transbronchoscopic biopy $(\mathrm{n}=1)$, paranasal sinuses and nasal biopsy $(n=14)$, percutanenous renal biopsy $(n=1)$, parotid gland biopy $(n=1)$ and skin biopsy $(n=1)$. The other 17 patients were diagnosed based on the 1990 American College of Rheumatology criteria (Table I) (9), when a minimum of 2 items in Table I were met. All patients received glucocorticosteroid therapy (predisone) at a dosage of $1.0 \mathrm{mg} / \mathrm{kg}$ per day for 4 weeks and then low dose maintenance. Following treatment, the symptoms of all patients was relieved to varying degrees. Written consent was obtained from all participants and the present study was approved by the Ethics Committee of the Hainan Branch of the Chinese PLA General Hospital (Hainan, China).

CT technique. In the present study, 43 patients underwent CT scanning. CT results were not available for the other 2 patients as they were transferred to a different hospital. CT scans were performed using a Siemens Sensation Cardiac 64 detector-row CT scanner (Siemens AG, Munich, Germany). The scan parameters were the same for each patient: Tube current, $900 \mathrm{~mA}$; tube voltage, $120 \mathrm{kV}$; slice thickness, $5.0 \mathrm{~mm}$; matrix, $512 \times 512$. The raw data were reconstructed using $5 \mathrm{~mm}$ thickness standard algorithms combined with a $2 \mathrm{~mm}$ thickness high-resolution construction in the region of interest. The images were mainly reviewed at the lung window setting with an about $1600 \mathrm{HU}$ of window width and a $600 \mathrm{HU}$ of window level. All the CT images were simultaneously reviewed by two independent and experienced chest radiologists who were blinded to the study conditions.

\section{Results}

Involved location of WG patients. System involvement varied between the 45 patients assessed in the present study and included thoracic involvement $(n=34)$, renal involvement $(n=10)$, skin involvement $(n=7)$, larger joint involvement $(n=14)$ and parotid gland involvement $(n=1$; data not shown). A total of 6 patients presented with the classic clinic triad (upper respiratory tract, lung and renal involvement).

Results of laboratory pathology investigation. Of the 32 patients who underwent ANCA screening examination (Table II), 27 (84.38\%) were ANCA-positive, which included cytoplasmic ANCA $(n=20)$ and perinuclear ANCA $(n=7)$. Among the 28 patients who underwent biopsy, 27 patients received a confirmed diagnosis $(96.43 \%)$.

Thoracic CT manifestations. A total of 34 patients presented with abnormal pulmonary CT findings (Table II), including large airway involvement $(n=1 ; 2.94 \%)$, abnormal lung findings $(n=31 ; 91.18 \%)$ and combined large airway and lung involvement $(n=2 ; 5.88 \%)$. Bilateral lung abnormalities were observed in 20 patients (58.82\%), unilateral lung abnormalities in 13 patients $(13 / 34,38.24 \%)$, pleural alterations combined with effusion in 4 patients $(11.76 \%)$ and pulmonary artery involvement in 2 patients $(5.88 \%)$.

The most common finding was pulmonary nodules ranging in size from $0.3-3.0 \mathrm{~cm}$, which were detected in 25 patients (73.53\%; Fig. 1), including unilateral pulmonary nodules $(n=7)$, solitary nodule $(n=3)$, ground-glass nodules $(n=2)$ and nodules combined with halo sign $(n=4)$. CT results for 14 patients revealed cavitary nodules and 5 patients presented with irregular cavity walls combined with necrotic debris (Fig. 2). Nodules combined with lobulation, speculation and vacuoles were observed in 5 patients and 1 patient presented with signs of vessel convergence. Additionally, 15 patients exhibited plaques $(44.12 \%)$ with ground glass opacity $(n=6)$, pulmonary consolidation $(n=6)$ and cavitation $(n=3$; Fig. 3$)$. Masses $(\geq 3 \mathrm{~cm})$ were observed in 6 patients $(17.65 \%)$ and were combined with cavitations in 4 patients, had fuzzy limits and irregular shape in 6 patients, and spiculation in 3 patients (Fig. 1). Bilateral pulmonary interstitial alterations were observed in 1 patient (Fig. 4).

Of the 34 patients who had abnormal pulmonary CT findings, severe airway alterations were observed in 3 (8.82\%). This includes 1 patient with changes in the vocal cord and subglottic region, 1 with intratracheal soft tissue shadow and another with bronchial wall thickening (Fig. 5). A total of 2 patients with pulmonary artery involvement were demonstrated to have undergone pulmonary artery alteration, including pulmonary artery wall thickening, which may lead to pulmonary artery embolism (Fig. 6).

Misdiagnosis based on CT findings. Of the 34 patients with clinically confirmed WG, 12 were diagnosed using CT results (35.29\%), 4 patients had inconclusive diagnoses (11.79\%), and 18 patients were misdiagnosed (52.94\%). Patient misdiagnoses were as follows: 12 were diagnosed with inflammation, including opportunistic infections in 1 patient, mixed infection in 1 patient, interstitial pneumonia in 1 patient, pneumonia with lymphocytic infiltrate in 1 patient, fungal infections in 5 patients and viral infection in 3 patients. The CT images obtained for patients who were misdiagnosed with inflammation featured ground glass opacity in 3 patients, patchy shadows in 8 patients, military nodules in 2 patients, pulmonary interstitial alterations in 1 patient, masses in 2 patients and pleural effusion in 2 patients. A total of 6 patients were misdiagnosed with tumors, including combined airway abnormality in 2 patients (airway thickening in 1 patient) and pulmonary interstitial alterations combined with fungal pneumonia in 1 patient.

In WG with lung involvement there may be various imaging manifestations. The atypical CT findings of WG were typically misdiagnosed as infectious lesions or neoplastic lesions. The irregular cavities were typically observed in patients with WG (Fig. 7).

CT imaging of pulmonary abscesses usually reveals thick-walled cavitations based on a high consolidation with the appearance of liquid-gas level in the cavity, which differs from WG. Fungal infections vary widely; floating bacteria may be observed in the void, which is not normally present in WG (16). WG may be misdiagnosed as primary pulmonary or pulmonary metastatic tumors with single and multiple solid mass or nodules (17). When WG appears as a single solid mass, it is not easily distinguished from other lung tumors using CT alone. There are certain differences between WG and pulmonary metastasis in CT findings of multiple nodules (18). It was observed in the present study that the multiple lung nodules of WG are typically shown as variable irregular shapes and the shadow connected to the lesion (Fig. 8). However, 
Table I. American college of rheumatology diagnostic criteria for Wegener granulomatosis.

$\begin{array}{ll}\text { Criteria } & \text { Characteristics }\end{array}$

Nasal or oral inflammation

Abnormal chest radiograph

Urinary sediment

Granulomatous inflammation at biopsy
Painful or painless oral ulcers, purulent or bloody nasal discharge

Nodules, fixed infiltrates, cavities

Microhematuria (>5 red blood cells per high-power field), red cell casts Involvement of the wall of an artery/arteriole, involvement of the perivascular/extra vascular space

The presence of two or more of the four criteria is associated with a sensitivity of $88.2 \%$ and a specificity of $92 \%$ for Wegener granulomatosis.

Table II. Clinical and CT manifestations of 45 patients with Wegener granulomatosis.

\begin{tabular}{|c|c|}
\hline Item & Value $(\%)$ \\
\hline \multicolumn{2}{|l|}{ General information } \\
\hline Male & $24(53.33)$ \\
\hline Female & $21(46.67)$ \\
\hline Age (years) & $46.4 \pm 12.2$ \\
\hline \multicolumn{2}{|l|}{ Clinical manifestation } \\
\hline Fever, combined ear/eye/mouth and nasal symptoms & $22(48.89)$ \\
\hline Fever, chest tightness, cough/cough blood & $6(13.33)$ \\
\hline Cough/cough sputum, ear/eye/mouth and nose symptoms, no fever & $13(28.89)$ \\
\hline Cough/cough/blood, no fever & $4(8.89)$ \\
\hline \multicolumn{2}{|l|}{ Laboratory examination } \\
\hline Positive ANCA & 27 \\
\hline Negative ANCA & 1 \\
\hline \multicolumn{2}{|l|}{ Thoracic CT findings ${ }^{\mathrm{a}}$} \\
\hline Nodules: Solid, ground glass opacity, cavity nodules & $11(25.58)$ \\
\hline Patchy shadows: Ground glass opacity, consolidation, combined cavity & $5(11.63)$ \\
\hline Lump, combined cavity & $1(2.33)$ \\
\hline Nodules combined with patch and/or lump & $12(27.91)$ \\
\hline $\begin{array}{l}\text { Intrapulmonary lesion combined with large airway involvement: diffused tube wall thickening } \\
\text { or intratracheal soft tissue }\end{array}$ & $2(4.65)$ \\
\hline Large airway involvement: Localized thickening of tracheal wall & $1(2.33)$ \\
\hline Intrapulmonary lesion combined with pulmonary artery: Tube wall thickening combined with embolism & $2(4.65)$ \\
\hline Normal thoracic CT findings & $9(20.93)$ \\
\hline
\end{tabular}

a2 patients did not undergo CT scan. ANCA, antineutrophil cytoplasmic antibody; CT, computed tomography.

multiple pulmonary metastases are generally located in the peripheral region of the lung, with a variable size and smooth boundaries.

\section{Discussion}

WG is a rare and angiogenic necrotizing vasculitis, involving the upper and lower respiratory tract, kidney and skin (19). The sex distribution (male:female) of WG is $3: 2$ and the peak incidence occurs in patients aged between 50 and 60 years (20). The incidence of WG has been increasing annually, which may be a result of increased environmental factors including cadmium, silica, mercury, sand dust and volatile hydrocarbons (9). WG may be a type of inflammation resulting from a hypersensitive response to external antigens (21). Trimethoprim-sulphamethoxazole has been previously used to successfully control the progression and relapse of WG (22).

Any part of the body may be involved in WG and the majority of patients present with classic clinic triad of the upper respiratory tract, lung and renal involvement (23). While the number of patients with renal involvement was low in the present study, the ratio of occurrence was lower in those who presented with the classic clinic triad. With the progression of the disease, $80-90 \%$ of patients with WG generally present with renal involvement (24). The clinical manifestations of WG are varied, a number of patients with WG present with 


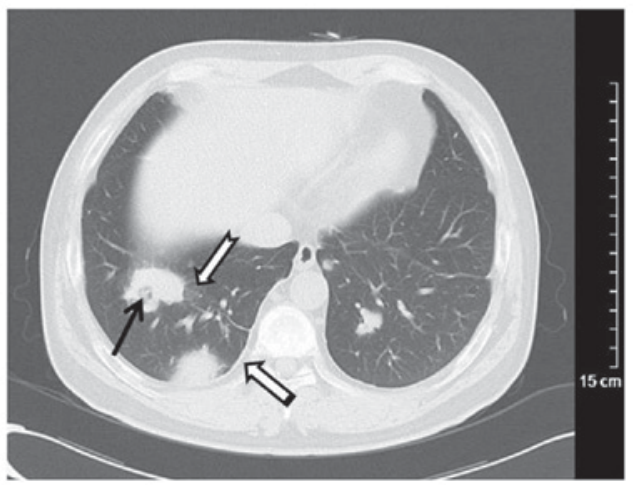

Figure 1. Wegener's granulomatosis in a 58-year-old female. Computed tomography scanning revealed multiple nodules and irregular masses in bilateral lungs, necrotizing cavities in the local lesions (black arrow), ground glass opacity in the outline of the lesion (dovetail arrow) and pleural thickening adjacent to the lesion (white arrow).
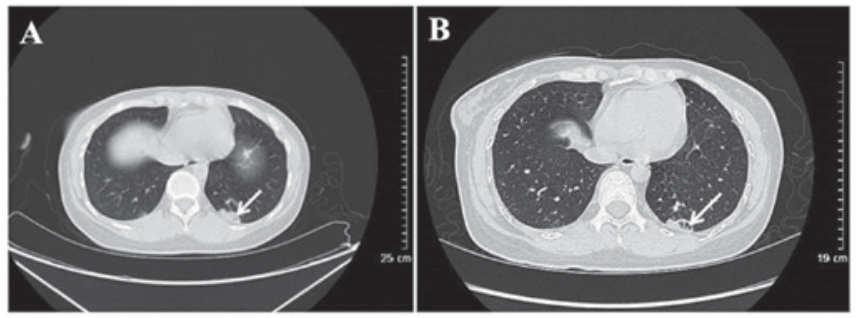

Figure 2. Wegener's granulomatosis in a 51-year-old female. Computed tomography scanning revealed (A) irregular nodules and cavitations in the left inferior lobar basal segment. (B) Following hormone treatment for 1 month (black arrow) the local lesion was reduced, necrotizing cavitations were larger and piecemeal necrosis was observed in cavitations (black arrow).

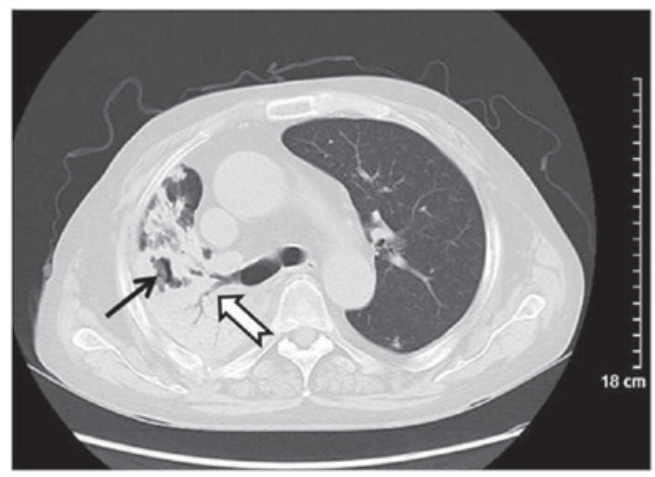

Figure 3. Wegener's granulomatosis in a 67-year-old male. Computed tomography scanning revealed lobar consolidation and irregular necrosis (arrows) in a large area of right lung.

long-term nasal congestion, which is often misdiagnosed as chronic sinusitis $(25,26)$. Furthermore, some patients present with acute renal and respiratory failure $(27,28)$. Patients with WG with thoracic involvement were typically present with cough, dyspnea, fever and chest pain (29).

WG has been reported to be a type of angiitis associated with ANCA, in particular cytoplasm ANCA (c-ANCA) (30). It was reported that the serum c-ANCA levels during the active phase of WG are elevated in $90 \%$ of patients (31). In the present study, $84.38 \%$ patients were ANCA-positive and $74.07 \%$

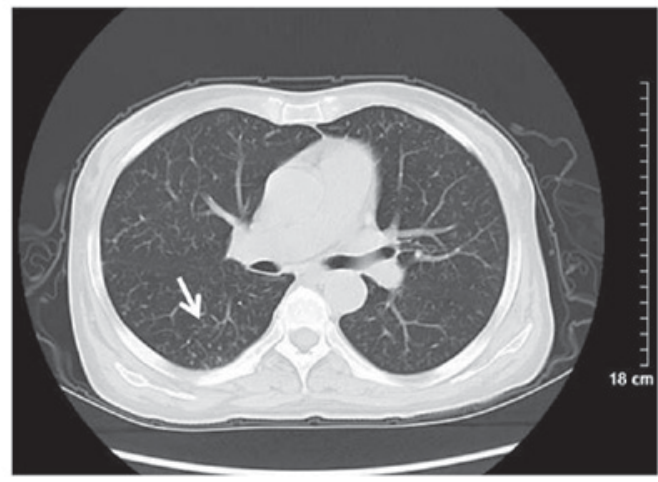

Figure 4. Wegener's granulomatosis in a 47-year-old female. Computed tomography scanning revealed diffuse small nodules with a centrilobular distribution (white arrow).

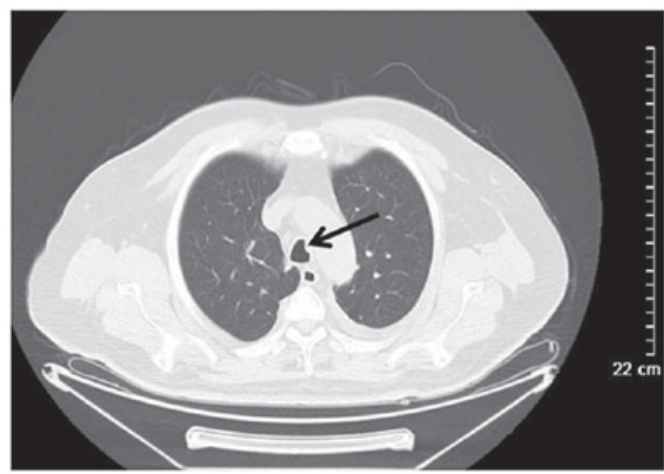

Figure 5. Wegener's granulomatosis in a 49-year-old woman. Computed tomography scanning revealed punctiform soft tissue density nodules in the main trachea (black arrow).

patients were positive for elevated c-ANCA levels (20/27); However other diseases, including polyarteritis nodosa, systemic lupus erythematosus, anaphylactoid purpura and Kawasaki disease may also present as c-ANCA-positive (9). Hence, biopsy is the gold standard in the diagnosis of WG (32).

As the majority of WG patients present with upper respiratory tract involvement and $90 \%$ of patients exhibit lung involvement (33), CT scans are essential for the diagnosis of WG. Multiple pulmonary nodules and masses are the most common manifestation of pulmonary involvement, the incidence rate of which was $40-70 \%$ in the present study. The rate of pulmonary masses was $17.65 \%(6 / 34)$ and the rate of nodules was $73.53 \%$ (25/34). Nodules were usually bilateral, numerous and scattered, ranging from $2-4 \mathrm{~cm}(15,34,35)$. The nodules in pulmonary involvement were similar, with signs of pulmonary tuberculosis, allergic reaction and acute bacterial, viral and fungal pneumonia with centrilobular distribution. The incidence of cavitation was $25 \%$ among patients with nodules $>2 \mathrm{~cm}(15,35)$. The walls of cavitations, which were eccentric or nodular, had varying thickness and were smooth or irregular. In the present study, the chest CT images revealed cavitation lesions with piecemeal necrosis in certain patients. Cavitations are susceptible to infection, leading to an increase in air-fluid levels, which is easily misdiagnosed as metastatic tumor, pulmonary abscess or septic pulmonary embolism (17). However, flocculent piecemeal fragment in the cavity of 


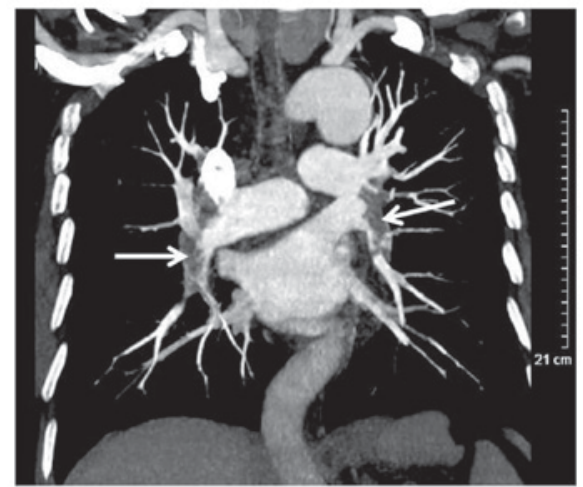

Figure 6. Wegener's granulomatosis in a 71-year-old male. Computed tomography scanning revealed a marked filling defect in bilateral pulmonary artery (white arrow).

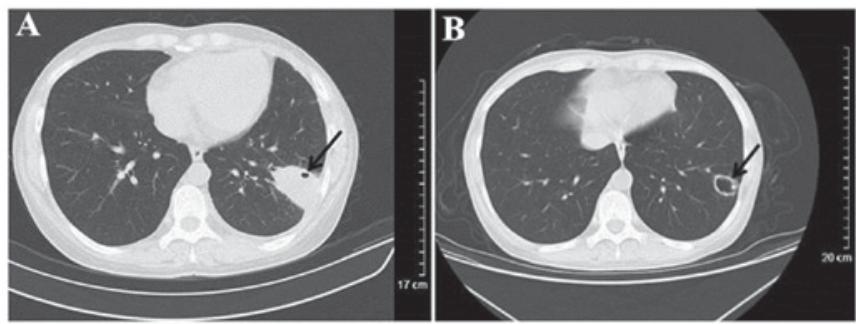

Figure 7. Computed tomography scanning of a patient with WG misdiagnosed as infectious lesions. (A) Irregular consolidation in the substrate out of left lower lung, and punctiform vesicle in the consolidation (black arrow). (B) At 3 months post predisone treatment, the lesion markedly reduced in size and evolved into a cavity with a thin-wall (black arrow).

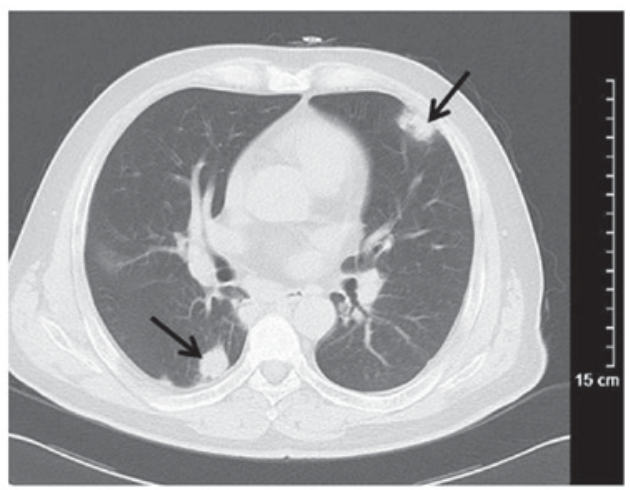

Figure 8. Computed tomography scanning of Wegener's granulomatosis misdiagnosed as tumor-like lesion. Nodules with irregular shapes were dispersed in the lung (black arrows).

metastatic cavity and pulmonary abscess is rare, which may be a useful CT sign for the differential diagnosis between metastatic cavity and pulmonary abscess. Hemorrhage surrounding the nodules manifested as a halo signal on CT images, which was visible at traumatic hemorrhage or alveolar exudation, including bronchioloalveolar carcinoma and abundant vascularity metastasis. In the present study, nodules with cavitation were observed in 14 patients, as indicated by increased air-fluid levels and uneven thickness of cavity walls. A total of 5 patients with nodules with cavitation were misdiagnosed, with a rate of $35.71 \%$; 4 patients were misdiagnosed with infection, and 1 was misdiagnosed with a tumor. Nodules with halo signals were observed in 4 patients. The lung parenchyma of patients with WG in the active stage with hemorrhage resulted in ground glass density and consolidation, which was misdiagnosed as pneumonia. The involvement of pulmonary arterioles in WG patents presented as mosaic perfusion and tree-in-bud (36). Ground glass density in the lungs of patents with WG may occur secondary to hemorrhages, exudation and mosaic perfusion of pulmonary alveoli in small vessel vasculitis (37). Pulmonary consolidation of patients with WG may be caused by pulmonary infarction or pulmonary infection due to small vessel vasculitis (38), which is similar to bacterial, viral and fungal pneumonia, pulmonary tuberculosis, pulmonary edema, acute respiratory distress syndrome or bronchioloalveolar carcinoma (39). A total of 15 patients presented with ground glass opacity and consolidation; 6 among them were misdiagnosed with different types of infection including opportunistic infection in one patient, lymphocytic interstitial pneumonia in another, tumor in 1 patient and fungal infection in 3 patients. Airway involvement has been identified as a late complication of WG, the incidence rate of which has been reported to be $15-25 \%$ (40). Airway involvement in WG has been misdiagnosed as tracheo-bronchial tumor and amyloidosis (39). The involvement of the heart and large vessels are rare manifestations of thoracic involvement in WG; 2 patients in the present study presented with damage to pulmonary arteries, which may be caused by the spread of granuloma. However, due to the incomplete information, there is a lack of sufficient evidence to support this point of view.

WG is frequently misdiagnosed as tumor-like lesions, including metastatic tumors and bronchial lung cancer. WG presenting with multiple solid nodules has been misdiagnosed as metastatic tumor; however, CT scans differ from those of patients with metastatic tumors (41). Multiple solid nodules of metastatic tumors are always variable in size and are mainly distributed in the peripheral lung, whereas multiple solid nodules of WG typically present with irregular outlines combined with smooth edges, as well as irregular nodules. CT scans of single nodules or masses in WG are also different in appearance to scans from patients with bronchial lung cancer (42). Additionally, the clinical manifestation and typical laboratory results for WG differ from tumor-like lesions (41). Hence, CT scans combined with clinical and laboratory analysis may reduce the rate of misdiagnosis.

Thorax involvement in WG is common and the CT manifestations are varied. The present study had several limitations, including the relatively small sample size. To improve the accuracy of the diagnosis of WG, it is necessary to perform a large number of case studies. Another shortcoming of the present study was the inconsistent diagnostic criteria. Of the 45 patients, only 28 cases were confirmed histologically, the other 17 cases were diagnosed clinically based on the American College of Rheumatology diagnostic criteria for Wegener granulomatosis. Additionally, another disadvantage was that the present study was retrospective and selection bias may affect the results.

In summary, WG is a rare disease with unknown etiology and complex clinical manifestation. Furthermore, due to difficulties with diagnosis and unfavorable prognosis, early diagnosis of WG is important to manage the symptoms of WG, 
prevent recurrence and improve the survival rate of patients Chest CT scans may be used as a basis for the primary diagnosis of WG. The CT manifestation of pulmonary involvement in WG was multitudinous, including multiple pulmonary nodules, ground glass opacity and consolidation, which were misdiagnosed as pneumonia, tumor and other diseases. The results of the present study suggest that multiple nodules and cavitations, irregular cavity walls and piecemeal necrosis in cavitations may be regarded as signs exclusive to WG. Hence, patients with the multiple solid nodules, ground glass opacity and consolidation may be diagnosed with WG. The exclusive signs combined with clinical and laboratory analysis, particularly negative ANCA, may be used as a basis for the diagnosis of WG. CT scans combined with clinical and laboratory analysis and biopsy may be required for the definite diagnosis.

\section{Acknowledgements}

Not applicable.

\section{Funding}

No funding was received.

\section{Availability of data and materials}

The datasets used and/or analyzed during the current study are available from the corresponding author on reasonable request.

\section{Authors' contributions}

JKL designed the study, CGL performed the experiments and JJL analysed the data.

\section{Ethics approval and consent to participate}

Written consent was obtained from all participants and the present study was approved by the Ethics Committee of the Chinese PLA General Hospital (Hainan, China).

\section{Consent for publication}

Not applicable.

\section{Competing interests}

The authors declare that they have no competing interests.

\section{References}

1. Wegener F: Über eine eigenartige rhinogene Granulomatose mit besonderer Beteiligung des Arterien systems und der Nieren. Beiträge Zur Pathologie 158: 127-143, 1976

2. Orden AO, Muñoz SA, Basta MC and Allievi A: Clinical features and outcomes of 37 Argentinean patients with severe granulomatosis with polyangiitis (Wegener Granulomatosis). J Clin Rheumatol 19: 62-66, 2013.

3. Bişkin S, Yazici ZM, Kayhan FT and Erdur Ö: Wegener Granülomatoziste KBB Tutulumu. KBB ve BBC Dergisi 17 62-65, 2009.

4. Rossini BA, Bogaz EA, Yonamine FK, Testa JR and Penido Nde O: Refractory otitis media as the first manifestation of Wegener's granulomatosis. Braz J Otorhinolaryngol 76: 541-541, 2010.
5. Scalcon MRR,Pereira IA, Filho AR and Paiva EDS: Manifestação otológica localizada em paciente com granulomatose de Wegener Localized otologic manifestation in a patient with Wegener's granulomatosis. Rev Bras Reumatol 48: 253-255, 2008.

6. Fonseca FP, Benites BM, Ferrari ALV, Sachetto Z, de Campos GV, de Almeida OP and Fregnani ER: Gingival granulomatosis with polyangiitis (Wegener's granulomatosis) as a primary manifestation of the disease. Aust Dent J 62: 102-106, 2017.

7. Bîrluţiu V, Rezi EC, Bîrluţiu RM and Zaharie IS: A rare association of chronic lymphocytic leukemia with c-ANCA-positive Wegener's granulomatosis: A case report. World J Surg Oncol 14: 145,2016

8. Gambini G and Leurini D: Cadmium exposure and Wegener's granulomatosis: Case report. Med Lav 83: 349-351, 1992.

9. Martinez F, Chung JH, Digumarthy SR, Kanne JP, Abbott GF, Shepard JA, Mark EJ and Sharma A: Common and uncommon manifestations of Wegener granulomatosis at chest CT: Radiologic-pathologic correlation. Radiographics 32: 51-69, 2012.

10. Wojciechowska J, Krajewski W, Krajewski P and Kręcicki T: Granulomatosis with polyangiitis in otolaryngologist practice: A review of current knowledge. Clin Exp Otorhinolaryngol 9: 8-13, 2016.

11. Olivencia-Simmons I: Wegener's granulomatosis: Symptoms, diagnosis, and treatment. J Am Acad Nurse Pract 19: 315-320, 2007.

12. Eustaquio ME, Chan KH, Deterding RR and Hollister RJ: Multilevel airway involvement in children with Wegener's granulomatosis: Clinical course and the utility of a multidisciplinary approach. Arch Otolaryngol Head Neck Surg 137: 480-485, 2011

13. Jbara MH, Vanlandingham A, Tawadros F and Moorman J: Cavitary lung lesion with pulmonary embolism: Case of active granulomatosis with polyangitis (Wegener's Granulomatosis). Poster Presentation, no.174. Appalachian Student Research Forum, D.P. Culp Centre at ETSU, Johnson City, TN, USA, April 6-7 2016.

14. Xia LL, Radiology DO and Hospital XR: Clinical evaluation onthe diagnostic value of multi-slice $\mathrm{CT}$ in the diagnosis of pulmonary Wegener's granulomatosis. Qiqihar Da Xue Xue Bao Yi Xue Ban 31: 19, 2015.

15. Guneyli S, Ceylan N, Bayraktaroglu S, Gucenmez S, Aksu K, Kocacelebi K, Acar T, Savas R and Alper H: Imaging findings of pulmonary granulomatosis with polyangiitis (Wegener's granulomatosis): Lesions invading the pulmonary fissure, pleura or diaphragm mimicking malignancy. Wien Klin Wochenschr 128: 809-815, 2016.

16. Bülbül Y, Ozlü T and Oztuna F: Wegener's granulomatosis with parotid gland involvement and pneumothorax. Med Princ Pract 12: 133-137, 2003.

17. Wang XL, Tan RS and Liu X: The CT manifestations analysis of pulmonary Wegener's granulomatosis with literature review. JPMI 16: 494-497, 2015.

18. Weir IH, Müller NL, Chiles C, Godwin JD, Lee SH and Kullnig P: Wegener's granulomatosis: Findings from computed tomography of the chest in 10 patients. Can Assoc Radiol J 43: 31-34, 1992.

19. Hanisch M, Fröhlich LF and Kleinheinz J: Gingival hyperplasia as first sign of recurrence of granulomatosis with polyangiitis (Wegener's granulomatosis): Case report and review of the literature. BMC Oral Health 17: 33, 2016.

20. Wallace ZS, Lu N, Miloslavsky E, Unizony S, Stone JH and Choi HK: Nationwide trends in hospitalizations and in-hospital mortality of granulomatosis with polyangiitis (Wegener's). Arthritis Care Res (Hoboken) 69: 915-92, 2017.

21. Gerhild TL, PMID and Wegener: Wegener'S Granulomatosis. Polic, 2012.

22. Kronbichler A, Jayne DR and Mayer G: Frequency, risk factors and prophylaxis of infection in ANCA-associated vasculitis. Eur J Clin Invest 45: 346-368, 2015.

23. White ES and Lynch JP: Pharmacological therapy for Wegener's granulomatosis. Drugs 66: 1209-1228, 2006.

24. Chen Y, Ding Y, Liu Z, Zhang H, Liu Z and Hu W: Long-term outcomes in antineutrophil cytoplasmic autoantibody-positive eosinophilic granulomatosis with polyangiitis patients with renal involvement: A retrospective study of 14 Chinese patients. BMC Nephrol 17: 101, 2016.

25. Ross JA, Mccune WJ and Sanders G: A 50-year old woman with nasal congestion, cough, and dyspnea. Allergy Asthma Proc 34: 188-192, 2013. 
26. Zycinska K, Straburzynski M, Nitsch-Osuch A, Krupa R, Hadzik-Błaszczyk M, Cieplak M, Zielonka TM and Wardyn K: Lund-mackay system for computed tomography evaluation of paranasal sinuses in patients with granulomatosis and polyangiitis. Adv Exp Med Biol 884: 13-19, 2016.

27. Kaya H, Yilmaz S, Sezgi C, Abakay O, Taylan M, Sen H, Demir M, Akkurt ZM and Senyigit A: Two cases of extrapulmonary onset granulomatosis with polyangiitis which caused diffuse alveolar haemorrhage. Respir Med Case Rep 13: 32-36, 2014.

28. Malavieille F, Page M, Ber CE, Christin F, Bonnet A and Rimmele T: The acute pulmonary renal syndrome: An unusual presentation of granulomatosis with polyangiitis. Rev Mal Respir 31: 636-640, 2014 (In French).

29. Kang CY, Liu CT, Wang YJ and Li TZ: Clinical data analysis and chest radiographic features of Wegener's granulomatosis with pulmonary involvement. Nan Fang Yi Ke Da Xue Xue Bao 30: 786-788, 2010 (In Chinese).

30. Gaffo AL: Diagnostic approach to ANCA-associated Vasculitides. Rheum Dis Clin North Am 36: 491-506, 2010.

31. Vega LE and Espinoza LR: Predictors of poor outcome in ANCA-associated vasculitis (AAV). Curr Rheumatol Rep 18: 70, 2016.

32. Kawakami T, Shimosaka R, Takeuchi S and Soma Y: Importance of appropriate location and frequency of biopsy for cutaneous manifestations in eosinophilic granulomatosis with polyangiitis. Int J Dermatol 55: 1388-1390, 2016.

33. Xu XL, Song W, Sui X, Song L, Qn DU and Wang X: Radiological and clinical features of eosinophilic granulomatosis with polyangiitis. Zhongguo Yi Xue Ke Xue Yuan Xue Ba 38: 617-620, 2016.

34. De Geeter F and Gykiere P: (18)F-FDG PET/CT imaging in granulomatosis with polyangiitis. Hell J Nucl Med 19: 5-6, 2016.
35. Feragalli B, Mantini C, Sperandeo M, Galluzzo M, Belcaro G, Tartaro A and Cotroneo AR: The lung in systemic vasculitis: Radiological patterns and differential diagnosis. Br J Radiol 89: 20150992, 2016.

36. Castañer E, Alguersuari A, Andreu M, Gallardo X, Spinu C and Mata JM: Imaging findings in pulmonary vasculitis. Semin Ultrasound CT MR 33: 567-579, 2012.

37. Mahajan V, Whig J, Kashyap A and Gupta S: Diffuse alveolar hemorrhage in Wegener's granulomatosis. Lung Indi 28: 52-55, 2011.

38. Lohrmann C, Uhl M, Kotter E, Burger D, Ghanem N and Langer M: Pulmonary manifestations of wegener granulomatosis: CT findings in 57 patients and a review of the literature. Eur J Radiol 53: 471-477, 2005.

39. Ananthakrishnan L, Sharma N and Kanne JP: Wegener's granulomatosis in the chest: High-resolution CT findings. AJR Am J Roentgenol 192: 676-682, 2009.

40. Rodrigues AJ, Jacomelli M, Baldow RX, Barbas CV and Figueiredo VR: Laryngeal and tracheobronchial involvement in Wegener's granulomatosis. Rev Bras Reumatol 52: 231-235, 2012 (In English, Portuguese).

41. Uppal S, Saravanappa N, Davis JP, Farmer CK and Goldsmith DJ: Pulmonary Wegener's granulomatosis misdiagnosed as malignancy. BMJ 322: 89-90, 2001.

42. Lynch JP III and Tazelaar H: Wegener granulomatosis (granulomatosis with polyangiitis): Evolving concepts in treatment. Semin Respir Crit Care Med 32: 274-297, 2011. 